\title{
Investigation of High Frequency Current Transformers Used for Partial Discharge Detection
}

\author{
S.H.HE ${ }^{1}$, Q.H.ZHAN ${ }^{1}$, S.B.HUANG ${ }^{1}$ \& J.B.WANG ${ }^{1}$ \&Y.M.ZHANG ${ }^{2}$ \& Y.P.XU ${ }^{2}$ \\ 1Guangdong Power Grid Corporation Foshan Power Supply Bureau, Foshan, China \\ ${ }^{2}$ Shanghai Jiao Tong University, Shanghai, China
}

KEYWORD: partial discharge; online monitoring; onsite detection; HFCT; frequency characteristics ABSTRACT: In the contribution, the equivalent circuit model of high frequency current transformers (HFCT), used for partial discharge online monitoring or onsite detecting, is established. Next, the major parameters of HFCT are derived and the influences on amplitude-frequency and phasefrequency characteristics are discussed. In the end, an optimized design for HFCT is proposed.

\section{INTRODUCTION}

Partial discharge monitoring inspects electrical equipment insulation state. Thus, on-line monitoring sensor shall meet the following requirements: easy installation, low noise, safety. The above advantages make HFCTs become one of the effective measures for on-line monitoring of the transformers, generators, power cables and other equipment partial discharges. The relationships between transfer function and various parameters are derived. On this basis, Optimization HFCT design presents.

\section{MODEL ANALYSIS}

HFCTs have similar structure with Rogowski coil (Fig.1).Partial discharge signal is small current signals. Thus, ultrachrystallite material having a high magnetic permeability is selected as HFCT core (inner diameter D1, an outer diameter D2, the height h), in order to improve sensitivity. In one direction by the winding core $\mathrm{N}$ turn coils and integrally connected to a resistor, usually close to the sampling circuit through a long coaxial cable. When the measurement happens, the current is measured by placing HFCT center. Figure 2 is a schematic diagram of a magnetic circuit analysis HFCT under common conditions. In the figure, il is measured currents; N1 is the turns (usually 1 ring) of to be measured current through the center of the current HFCT. U1 is the voltage to be measured by the HFCT coupled to the circuit. The secondary winding is HFCT output coil, i2 is the output current coupled by the HFCT. The secondary coil of HFCT (integral characteristic impedance of the coaxial cable resistance should be) is a coaxial cable, which is a few to tens of meters long connection to the integrator resistor. It corresponds to CL and RL (integrating resistor) connected in parallel, as shown in FIG. 2.

Then, working mechanism HFCTs magnetic field will form a view for analysis. Magneto motive force (MMF) F1, F2 are generated by the primary and secondary coil :

$$
\begin{aligned}
& F_{1}=\oint \overrightarrow{H_{1}} \cdot d \vec{l}=N_{1} \cdot i_{1} \\
& F_{2}=\oint \overrightarrow{H_{2}} \cdot d \vec{l}=N_{2} \cdot i_{2}
\end{aligned}
$$

the primary coil and the secondary coils generate the magnetic fields $\overline{H_{1}}$ and $\overline{H_{2}}$.

At this time, the remaining MMF system.

$$
F_{m}=F_{1}+F_{2}=N_{1} \cdot i_{m}
$$

Where in, im is converted current, which generates FM, to the primary side. From Eq. (1), (2) and (3) it can be deduced 


$$
\left(i_{1}-i_{m}\right)+\frac{N_{2}}{N_{1}} \cdot i_{2}=0
$$

From Eq. (4), we see that the smaller $i_{m}$ will make ratio of the primary current to the secondary more linear. Therefore, we reduce can to improve accuracy.

According to the principle of electromagnetic induction, HFCT U2 voltage induced in the secondary side is

$u_{2}=\frac{d \Psi_{m}}{d t}=N_{2} \cdot \frac{d \Psi_{m}}{d t}$

Acquire the relationship of $\mathrm{u}_{2}$ and $i_{m}$ from Eq.(3) and (5):

$u_{2}=\frac{N_{1} N_{2}}{\mathfrak{n}} \cdot \frac{d i_{m}}{d t}$

श) is the core reluctance of the HFCT . Perform Fourier transform on Eq. (6):

$U_{2}=j \omega \cdot \frac{N_{1} N_{2}}{\Re} \cdot I_{m}$

From Ohm's Law, obtain the relationship between $U_{2}$ and $I_{2}$ :

$U_{2}=-I_{2} /\left(j \omega C_{L}+\frac{1}{R_{L}}\right)$

Substitute Eq. (7) and (8) into Eq. (9), current $I_{1}$ the transfer function to the voltage $U_{2}$ is:

$H(j \omega)=U_{2} / L_{1}=\frac{N_{1}}{N_{2 L}} \cdot\left(\frac{1}{\frac{1}{R_{L}}+j \omega C_{L}+\frac{1}{j \omega L_{L}}}\right)$

Where $L_{L}=\frac{N_{2}^{2}}{\mathrm{~N}}$. In Eq. (9), Section is very similar to a parenthesis RLC parallel resonance circuit with resistance $R_{1}$, capacitors and inductors $C_{1} . L_{1}$. Therefore, we can analyze HFCT performance using a parallel RLC resonant circuit theory.

Slightly modify the Eq. (9), we can get:

$H(j \omega)=\frac{N_{1}}{N_{2}} \cdot\left(\frac{R_{Z}}{1+\left(\frac{j \omega}{\omega C}+\frac{w_{C}}{j \omega}\right) \ell}\right)$

The center frequency $\omega_{C}=\frac{1}{N_{2}} \cdot \sqrt{\frac{\mathrm{N}}{C_{L}}} ; Q=R_{L} \sqrt{\frac{C_{L}}{L_{L}}}=\frac{R_{L}}{N_{2}} \cdot \sqrt{\mathrm{N} C_{L}}$ is the quality factor, which are two important parameters affecting HFCT performance.

According to Eq. (10) the amplitude- and phase-frequency characteristics of $H(j \omega)$ can be calculated:

1) A HFCT transfer function is a first-order band-pass filter, the center angular frequency is $\omega_{C}$. It meets:

$$
\begin{gathered}
\begin{cases}|H(j \omega)|=\frac{N_{2}}{N_{2}} \cdot R_{L} & \left(\omega=\omega_{C}\right) \\
|H(\omega)|<\frac{N_{1}}{N_{n}} \cdot R_{L} & \left(\omega \neq \omega_{C}\right)\end{cases} \\
\angle \mathcal{H}(j \omega)= \begin{cases}>0 & \left(\omega<\omega_{C}\right) \\
=0 & \left(\omega=\omega_{C}\right) \\
0 & \left(\omega>\omega_{C}\right)\end{cases}
\end{gathered}
$$


2) There is close relationship between the lower cutoff frequency $\omega_{L}$ and the higher cut-off frequency $\omega_{H}$ of the transfer function and Q:

$$
\begin{aligned}
& \omega_{L}=\frac{2}{\sqrt{Q^{-2}+4}+Q^{-1}} \cdot \omega_{C} \\
& \omega_{H}=\frac{\sqrt{Q^{-2}+4}+Q^{-1}}{2} \cdot \omega_{C}
\end{aligned}
$$

In Fig. 3, the amplitude and HFCT transfer function, which can be seen that the phase - frequency curve: (1) when the angular frequency of $\omega_{C}$, the transfer function reaches its maximum amplitude $\frac{N_{1}}{N_{2}} \cdot R_{L}$ with a $20 \mathrm{~dB} / \mathrm{dec}$ attenuation slope; (2) The smaller Q is, transfer function is in a wider pass-band, better phase - frequency characteristics. This is a great validation Eq. (11) to (14).

In short, a HFCT transfer function is a first-order band pass filter. The two main parameters that affect its performance is the center frequency $\omega_{C}$ and quality factor Q. We can widen the HFCT pass band by reducing $\mathrm{Q}$ to improve performance. We know $Q=\frac{R_{L}}{N_{2}} \cdot \sqrt{\mathrm{N} C_{L}}$, so we can reduce Q by: (1) reducing $N$, (2)reducing $C_{L}$, (3)reducing $\frac{R_{L}}{N_{2}}$ 。
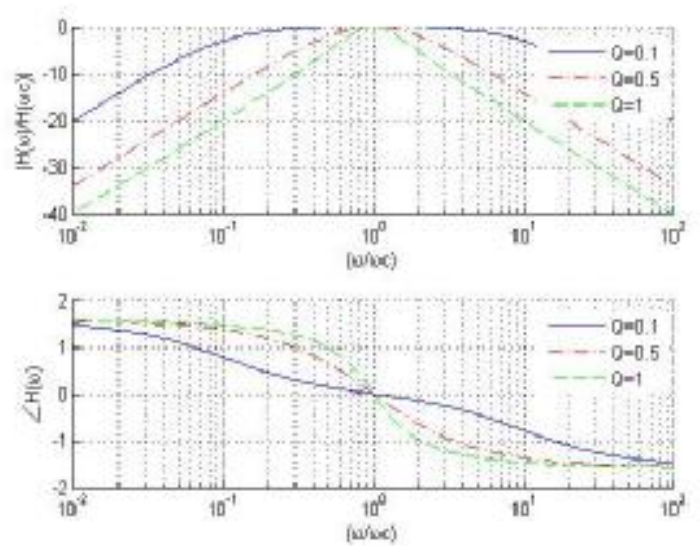

Fig.1 the relationship between the amplitude-frequency and phase-frequency characteristics and Q of $H(j \omega)$

1) Reducing $N$

Toroidal cores without air gap reluctance dimensions in Figure 1 is

$$
Y^{Y}=\frac{2 \pi}{\mu \mu_{0} h \cdot \ln \left(D_{2} / D_{2}\right)}
$$

Where $\mu_{0}$ the permeability of air is, $\mu$ is the relative permeability of the core material. As can be seen, if there is no air gap reluctance annular core $\mathrm{N}^{\prime}$ is inversely proportional to its relative permeability $\mu$. The $\mu$ values of magnetic materials are nonlinear. They change because of changes in magnetic flux density. It is very possible that this core is made so that the third harmonic HFCTs into the test results even penetrate the core. This is unacceptable HFCTs and must take measures to curb this phenomenon.

Typically, the gap is cut off to improve the linearity of the core. Having an air gap in size as shown in Fig. 1 toroidal core, whose length is $\delta$ Magnetic field analysis is shown in Fig.4. According to Maxwell's equations, you can understand, the magnetic flux density B(r) of the radius $r$ is the same gap in the air. The relationship between B(r) and MMF F meets: 
$F=\frac{B(r)}{\mu_{0}} \delta+\frac{B(r)}{\mu \mu_{0}}(2 \pi r-\delta)$

core reluctance $N$ is:

$\mathfrak{R}=\frac{2 \pi}{\mu \mu_{0} h \cdot \ln \left(\frac{\pi D_{2}+(\mu-1) E^{2}}{\pi D_{1}+(\mu-1)}\right)}$

By comparison, we find that the Eq. (17) in the general form of Eq. (15). When $\delta \gg \frac{\pi D_{2}}{(\mu-1)}$, Eq. (17) can be:

$\eta \frac{\bar{\delta}}{\mu_{0} \cdot s}$

The $s$ is a cross-sectional area of the core. At this point, the permeability $\mu$, nonlinear may introduce been eliminated from the equation. (18).

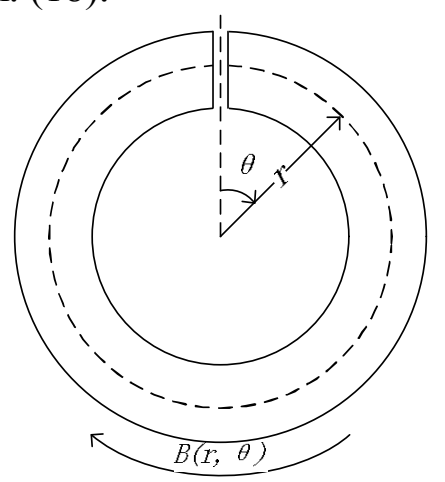

Fig. 2 Core magnetic field analysis

From (17), the core reluctance $N$ increases with the air gap width $\delta$. The smaller $\delta$ and $N$, the better HFCT is performance. However, considering linearity, the gap length $\delta \gg \frac{\pi D_{2}}{(\mu-1)}$ has been selected to suppress non-linearity.

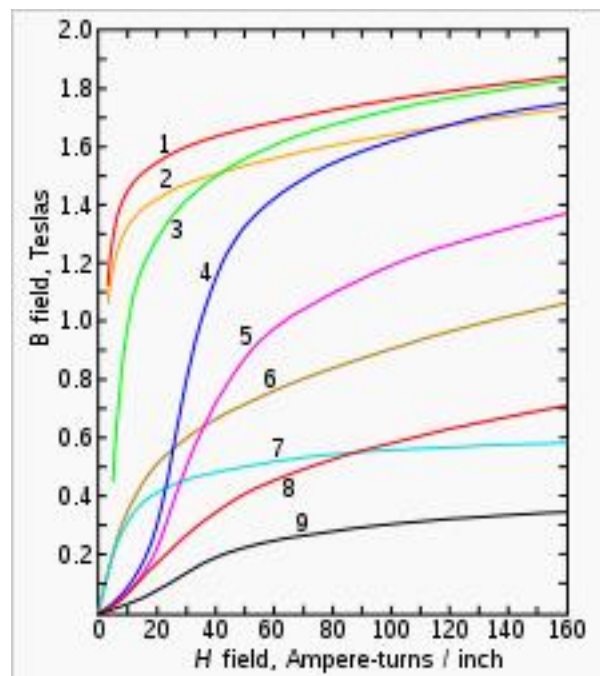

Fig. 3 magnetic saturation curve

$N$ reduce the problems caused by the increase in $\delta$ can be obtained by increasing the core crosssectional area $\mathrm{s}$ to compensate. Increasing $\mathrm{s}$ is also to prevent magnetic saturation. Figure 5 is a graph of magnetic saturation of various ferromagnetic material, can be seen, there is saturation magnetic induction $B_{S}$ of variety of ferromagnetic materials. Magnetic induction hardly increases after the core reaches the $B_{S}$. While the partial discharge signal is typically low, current signal does not cause HFCT saturation, there is all some of the leakage capacitance of the electrical equipment, 
when it works at its rated voltage, a number of power-frequency current to be introduced to the object being measured. It can cause very easy to be saturated, if not handled properly.

For example, the measured maximum power frequency component of the current is $\boldsymbol{I}_{\max }$, the magnetic induction in the core is $\mathrm{B}$, following relationships are met:

$U_{2}=\frac{N_{1}}{N_{2}} \cdot I_{\max } \cdot R_{\mathrm{L}}=j \omega N_{2} \cdot B \cdot s$

A general magnetic material satisfies $B<B_{S} / 2$, when working, whereby the core crosssectional area constraint can be obtained:

$s>\frac{N_{1}}{50 \pi N_{2}^{2}} \cdot \frac{s_{\max }}{B_{S}} R_{L}$

2) Reducing $C_{L}$

$C_{L}$ is HFCT coil drain capacitance. It mainly consists of two parts - the coil and the output terminal, and its coil wire lead leakage capacitance between the leakage capacitance.

$C_{L}$ impendence becomes small when the signal frequency is high, thus affecting HFCT response sensitivity. Through theoretical analysis, we can know the parasitic capacitance of the coil is proportional to the length of the coil. Thus, in the production of coils, minimizing the length of the wire between the output terminal, and the core may be designed to have a square cross-section, so as to minimize the coil retaining a constant cross-sectional area of the length.

3) Reducing $\frac{R_{L}}{N_{2}}$

Partial discharge monitoring of high voltage equipment insulation condition is detected. Generally, HFCT attached to the device under test. In power cables, for example, the wavelength by the partial discharge occurs in the body of the discharge signal is usually about 30 meters. It is the same length as the magnitude of the coaxial cable. Thus coaxial cable distribution parameters and the following circuit must be considered. So integrating resistor RL must match the coaxial cable (usually $50 \Omega$ ) of the characteristic impedance. Only in this way, the "coaxial cable + integral resistance" structure can be equivalent to a lumped resistance $R_{L}$

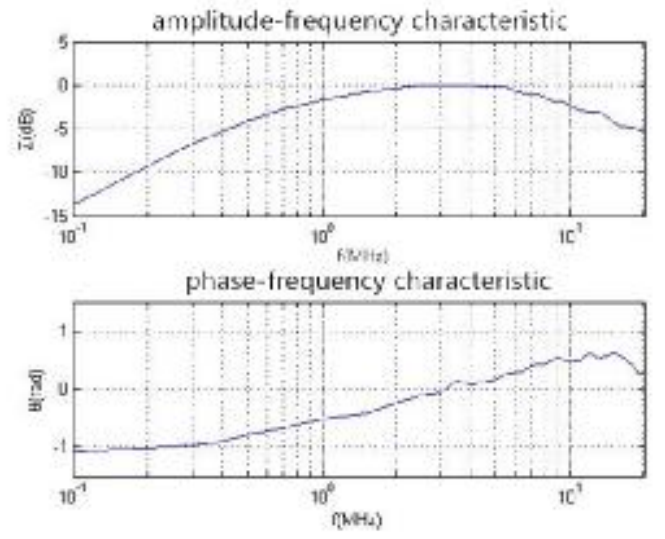

Fig.4 HFCT Frequency Characteristic Diagram

And it can increase N2 to reduce Q. But we also need to note: the HFCT transfer function in the amplitude of the center frequency of $\frac{N_{1}}{N_{2}} \cdot R_{L} \cdot N_{2}$ increasing will reduce the sensitivity of the reaction, which is on-line monitoring system for partial discharge is very important. A lot of interference present in the operating environment of electrical equipment and partial discharge signal is very subtle. If insufficient sensitivity, signal to noise ratio of the system (SNR), will be significantly reduced. Therefore, the discharge signal or even is buried in a complex electromagnetic environment. 
Although the $N_{1}$ increase may make up for the increase $N_{2}$ defects to a certain extent, this also increases the HFCT power frequency components. This will inevitably lead to the core in a higher operating point, making it more possible to be more saturated (see Eq. (20)).

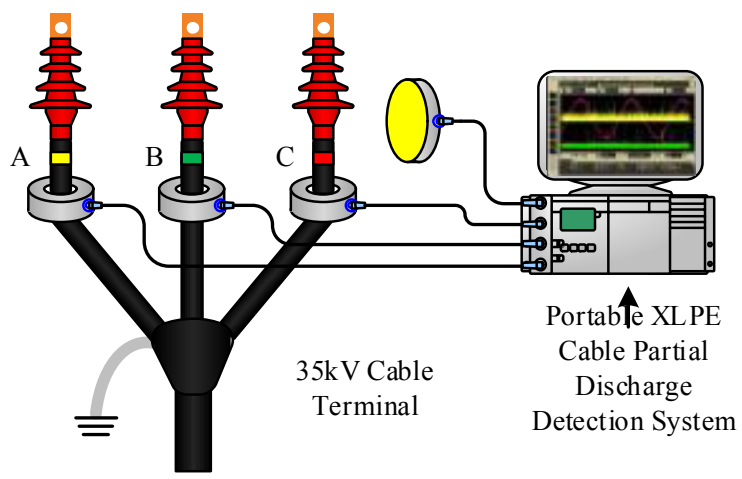

Fig.5 Field Test Schematic

$\mathrm{T}$ Therefore, adjusting the number of turns designed to improve the performance of only the accurate calculation (core saturation) weighed (sensitivity and the pass-band) and after take.

\section{EXPERIMENT DATA}

After the theoretical calculation, a HFCT (see Figure 6 is a frequency characteristic) of $35 \mathrm{kV}$ and below XLPE cable line partial discharge detection is designed. It is tested to $35 \mathrm{kV}$ XLPE cable terminal substation partial discharge conditions. UHF sensor is used to help verify the test results.

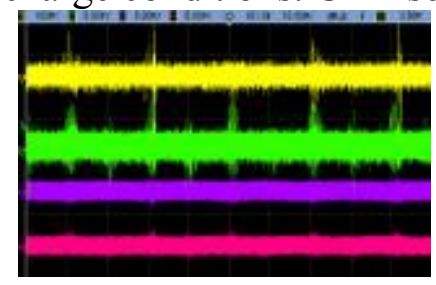

(A) Multi-Period Screenshot

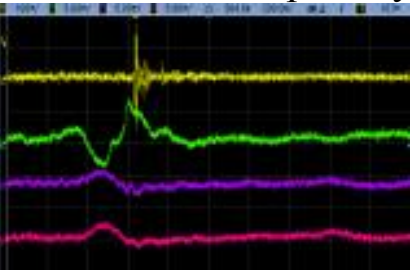

(B) PD pulse waveform

Fig.6 Test Results

Figure 7 shows a schematic view of the cable terminal substation test: place near the end of the cable, and the three HFCTs to the body on the cable terminals A, B and C of the ultra-high frequency sensor. 4 sensor output signals is connected to $\mathrm{CH} 1$ "portable cable partial discharge detection system", respectively, after installation, the output signal of each sensor of methane observed on "detection system" screen.

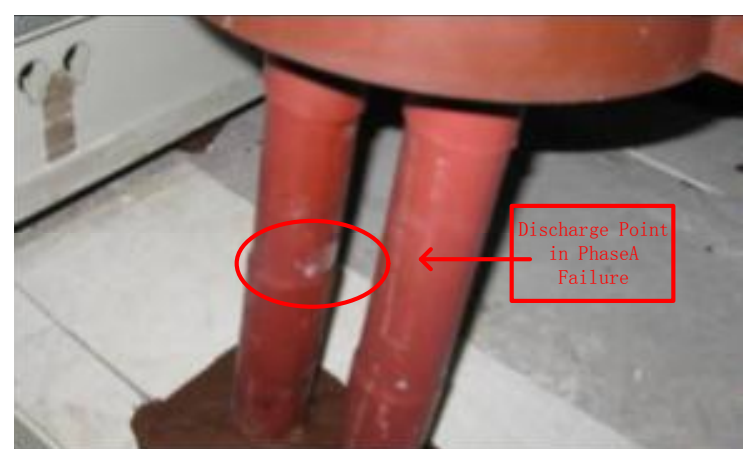

Fig.7 discharge source

Figure 8 is the test results. From top to bottom is the ultra-high frequency signals and highfrequency current signal cable phase A, B and C of the body. From (a), and can be seen in HFCT UHF sensor and detected phase A significant discharge pulse has a significant cyclical characteristics. From (b), phase A HFCT phase discharge measurement signal is approximately $5 \mathrm{MHz}$; in stage B and $\mathrm{C}$ simultaneously detect signals of the same frequency of the discharge, but small, and $\pi$ amplitude ratio A phase retardation. 
It is comprehensive analysis of this issue. It is believed that there are in some of the cable end of the discharge and the main discharge power is in phase A. The cable holder was opened. Significant fault discharge traces was found on the cable phase A (Fig. 9) body.

\section{CONCLUSION}

In this article, we have HFCTs modeling and analysis. The following conclusions:

1) Reduce the core reluctance $\mathrm{N}$ expand HFCT pass-band. Toroids reluctant, this core production HFCTs is serious nonlinear. We can reduce HFCT nonlinearity by cutting an air gap of width $\delta$ to satisfy $\delta \gg \pi D_{2} /(\mu-1)$.

2) A gapped core's reluctance

$N$ is proportional to the gap width $\delta$ and inversely proportional to the cross-sectional area S. Small $\delta$ can cause HFCT saturation. Then, we should increase $\mathrm{S}$ to suppress such saturation.

3) The HFCT pass band can also be improved by reducing the leakage capacitance $C_{L}$ of the coil. A square cross-section is designed to minimize the length of the coil, reducing $C_{L}$

4) Integral resistor must match the characteristic impedance of the coaxial cable, to prevent reflection HFCT output signal, which can interfere with the measurement.

Finally, the sensor is a testament to experimental verification, in partial discharge monitoring good performance.

\section{ACKNOWLEDGMENT}

This work was supported by the Guangdong Power Grid Corporation (K-GD2013-047), The research project of field acceptance and operation maintenance's tests and detection methods of medium voltage cable.

\section{REFERENCES}

[1] DAI Wei, TANG Xiu-song, High-frequency Pulse Current Method Applied in Partial Discharge of Online Detection of Power Transformer [J]. Automation Application, 2014, (12).

[2] DU You-wei. The Development of Magnetic Materials[J]. physics, 2000, 29(6):323-332.

[3] CHEN Teng-biao, WU Tao, WEI Qian-hu, et al. Application of High Frequency Pulse Current Method in Detecting and Positioning for High Pressure Cable Electrification Partial Discharge[J]. Guangdong Electric Power, 2014, (1):114-119.

[4] GU Jiang-guo, WANG Shi-ping, SUN Xiao-qian. Property and Development of Normal Dielectric Insulating Material [J]. Chemical Engineer, 2002, (4):54-55. 